\title{
An exact equilibrium model of an unbound stellar system in a tidal field
}

\author{
M. Fellhauer ${ }^{1}$ and D. C. Heggie ${ }^{2}$ \\ 1 Sternwarte Universität Bonn, Germany \\ e-mail: mike@astro.uni-bonn.de \\ 2 School of Mathematics, University of Edinburgh, Scotland, UK \\ e-mail: d.c.heggie@ed.ac.uk
}

Received 22 December 2004 / Accepted 9 February 2005

\begin{abstract}
Star clusters and dwarf galaxies gradually dissolve as they move in the potential of their host galaxy. Once their density falls below a certain critical density (which is comparable with the background density of the galaxy) it is often assumed that their evolution is completed. In fact the remnant of such a system forms a distribution of stars which are unbound to each other and which move on similar orbits in their host potential. With this motivation we study the evolution of an idealised unbound system and follow its expansion and dissolution in the tidal field of a model galaxy. Initially the stars are uniformly distributed (with a density below the critical density) within an ellipsoidal volume. The system itself travels on a circular orbit within a galaxy modelled as an isothermal sphere. The initial velocities of the stars are chosen by assuming that they move on (three-dimensional) epicycles with guiding centre at the centre of the ellipsoid, though the usual epicyclic theory is altered to account for the self-gravity of the system. This is believed to be the first exact equilibrium model of a stellar system in a tidal field. Our main task is to study the stability of such configurations and the time-scale of their dissolution, as a function of the initial density and size of the ellipsoid. If the time of dissolution is measured by an increase of the half-mass radius of $50 \%$, we find that systems of low density ( $\sim 1 \%$ of the background density) and small radius (50 pc on an orbit of radius $10 \mathrm{kpc}$ ) can survive for about 20 galactic orbits. For small systems we show that the lifetime is approximately proportional to the inverse square root of the density.
\end{abstract}

Key words. galaxies: star clusters - methods: $N$-body simulations - Galaxy: kinematics and dynamics

\section{Introduction}

In recent years, considerable effort has been spent in examining the destructive processes which lead to the dissolution of star clusters and dwarf galaxies, and the influence of these processes on the distribution function of these objects (e.g. Baumgardt 1998) and on the host galaxy. There are two main areas of interest. First there is the dissolution caused by internal processes such as evaporation (e.g. Giersz \& Heggie 1997; Baumgardt \& Makino 2003). The second issue is the merging of these objects with the body of the galaxy (e.g. Velazquez \& White 1999) or with each other (Fellhauer et al. 2002). Within the context of the first problem, investigations usually stop when the object has dissolved according to some tidal stability criterion (Sect. 2). So far little attention has been paid to their subsequent fate, i.e. what happens to these objects after they become unbound entities.

Retrograde motions are expected to play an important role in these unbound systems. It has been known for a long time (Hénon 1969) that stars in retrograde motion about a star cluster (at least for clusters on circular galactic orbits) are stable (i.e. orbitally stable) even outside the tidal boundary; their orbits are best thought of as perturbed epicycles. As a star cluster loses mass and its tidal radius shrinks, we may expect that it is surrounded by some stars moving approximately on such orbits.

Based on these considerations, the present study starts with a very special toy model in which all the stars move on retrograde orbits resembling epicycles with a common guiding centre. Their spatial distribution is a homogeneous ellipsoid, and the centre of the ellipsoid moves on a circular orbit within the potential of an isothermal sphere, which is taken as a model of the host galaxy. The simplicity of our setup permits some analytical investigation which gives useful insights into the results of numerical simulations. It is also believed to be the first example of an exact equilibrium of a stellar system in a tidal field.

Having set up the sequence of models, our main interest turns to their stability. In particular, we aim to investigate the time-scales on which they evolve and finally disperse, and their spatial and kinematic properties as they do so.

In the next section we briefly go through the theory of epicyclic motion with modifications to account for the selfgravity of the system itself. We also explain the setup of our toy model. Then we describe the results of our simulations, which cover a large parameter space. Finally we give our conclusions, 
and discuss their possible relevance for real systems, such as those found in and around our Milky Way.

\section{Theory and setup}

The basic idea of our model is the following. Inside an ellipsoid of uniform density in a linear tidal field, orbits are modified epicycles, with a harmonic motion in $z$ of amplitude $z_{0}$, say (cf. Fig. 1). We consider epicycles which are centred at the centre of the ellipsoid. For a given epicyclic amplitude, we construct a distribution of $z_{0}$ so that the space density of this superposition of epicycles is independent of $z$ (up to the edge of the ellipsoid). Then we construct a distribution of epicyclic amplitudes so that the space density is also uniform in $x$ and $y$. Because we have arranged for the amplitude of the epicyclic $x$ - and $y$-motions to be in the same proportion as the corresponding axes of the ellipsoid, we can build up the entire ellipsoid in this way.

In principle this gives rise to an equilibrium model. Our $\mathrm{N}$-body simulations, however, do not maintain equilibrium because of relaxation, because the orbits are dynamically unstable, and because the $N$-body simulations use the exact tidal field and not a linear approximation.

Let $X, Y, Z$ be galactocentric coordinates, with $Z$ perpendicular to the plane of motion of the system under study, and let $R^{2}=X^{2}+Y^{2}$. Epicyclic theory is a linearised approximation in which stars move in a potential

$\Phi=\left.\frac{1}{2} \frac{\partial^{2} \Phi_{\mathrm{eff}}}{\partial R^{2}}\right|_{D, 0}(R-D)^{2}+\left.\frac{1}{2} \frac{\partial^{2} \Phi_{\mathrm{eff}}}{\partial Z^{2}}\right|_{D, 0} Z^{2}$

(Binney \& Tremaine 1987), where $D$ denotes the distance to the axis of symmetry of the galaxy and $\Phi_{\text {eff }}(R, Z)$ is the effective galactic potential. We set

$$
\begin{aligned}
& \left.\frac{\partial^{2} \Phi_{\mathrm{eff}}}{\partial R^{2}}\right|_{D, 0}=\kappa^{2}, \\
& \left.\frac{\partial^{2} \Phi_{\mathrm{eff}}}{\partial Z^{2}}\right|_{D, 0}=v^{2},
\end{aligned}
$$

where $\kappa$ is the epicyclic frequency. For the potential of a singular isothermal sphere these frequencies are given by

$$
\begin{aligned}
& \kappa=\sqrt{2} \Omega, \\
& v=\Omega,
\end{aligned}
$$

where $\Omega=V_{0} / D$ is the angular velocity of motion on a circular orbit of radius $D$ around the host galaxy and $V_{0}$ is the circular velocity.

We adopt initial conditions in which stars are distributed uniformly within an ellipsoid with semi-major axes $a, \xi a, a$, where $a>0$ is a free parameter, and $\xi>1$ will be determined from the shape of closed epicycles. Therefore we have to take the potential of a prolate ellipsoid of uniform density $\rho$ into account. We use rotating, cluster-centered coordinates $x$, $y, z$ with origin at the centre of the ellipsoid of stars. The coordinate axes are directed away from the galactic centre, in a direction opposite that of the circular motion round the galaxy,

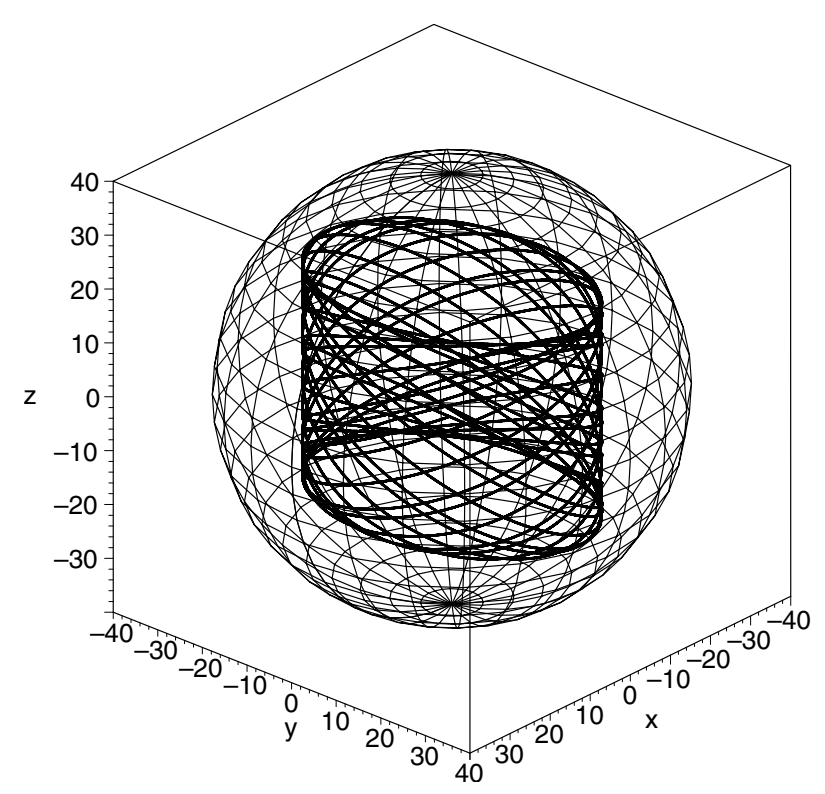

Fig. 1. A schematic representation of a uniform ellipsoid and one orbit.

and orthogonal to the plane of motion, respectively. The appropriate differential equations in the epicyclic approximation are then (Chandrasekhar 1942)

$\ddot{x}+2 \Omega \dot{y}+\left(\kappa^{2}-4 \Omega^{2}\right) x=-B_{1} x$

$\ddot{y}-2 \Omega \dot{x}=-B_{3} y$

$\ddot{z}+v^{2} z=-B_{1} z$.

Here, $B_{1}=2 \pi G \rho A_{1}$ and $B_{3}=2 \pi G \rho A_{3}$, where $A_{1}$ and $A_{3}$ are given by standard formulae for the potential of a homogeneous prolate ellipsoid (Binney \& Tremaine 1987, Table 2-1).

This additional acceleration alters the frequencies of motion and the shape of closed epicycles. Eigensolutions of these equations give frequencies $\kappa^{\prime}$ and $v^{\prime}$ defined by

$\kappa^{\prime 2}=\frac{1}{2}\left(\left(\kappa^{2}+B_{1}+B_{3}\right) \pm \sqrt{\left(\kappa^{2}+B_{1}-B_{3}\right)^{2}+16 \Omega^{2} B_{3}}\right)$

$v^{\prime 2}=v^{2}+B_{1}$

and the epicyclic ratio

$\xi=\frac{\kappa^{\prime 2}+\kappa^{2}-B_{1}}{2 \Omega \kappa^{\prime}}=\frac{2 \Omega \kappa^{\prime}}{\kappa^{\prime 2}-B_{3}}$.

Note incidentally that the two expressions for $\xi$ in Eq. (11) are identical if Eq. (4) and the upper sign in Eq. (9) are used.

If $|\kappa| \gg B_{1}, B_{3}$, then Eq. (9) implies that $\kappa^{\prime} \simeq \kappa$ (if we choose the upper sign), and Eq. (11) gives approximately the familiar axial ratio of epicyclic motion. In the general case, since we also use $\xi$ for the axial ratio of the ellipsoid, the right side of Eq. (11) depends on $\xi$. Therefore we have solved for $\kappa^{\prime}$ and $\xi$ using the above equations iteratively (taking the upper sign in Eq. (9)).

A second solution of the differential equations (Eqs. (6) and (7)) is obtained if one takes the lower sign for $\kappa^{\prime}$. It leads to an imaginary frequency $i k=\kappa^{\prime \prime}$ provided that

$\rho<\frac{4 \rho_{0}}{A_{1}}$ 
where $\rho_{0}$ denotes the density corresponding to the isothermal background. (This is always fulfilled in our parameter range, though there is a series of bound systems (i.e. systems not satisfying Eq. (12)) where all frequencies are real.) We can see that $k \ll \kappa^{\prime}$ if $\rho$ is much smaller than the critical density (cf. Eq. (12)), and then we have:

$$
\begin{aligned}
k^{2} & =-\frac{1}{2}\left(\left(\kappa^{2}+B_{1}+B_{3}\right)-\sqrt{\left(\kappa^{2}+B_{1}-B_{3}\right)^{2}+8 \kappa^{2} B_{3}}\right) \\
& \approx B_{3} \\
\xi^{\prime} & =\frac{2 \Omega k}{k^{2}+B_{3}} \\
& \approx \frac{\Omega}{\sqrt{B_{3}}},
\end{aligned}
$$

in which $\xi^{\prime}$ is the ratio of the components of the corresponding eigenvector.

Within the epicyclic approximation the complete solution is a linear combination of both solutions, i.e.

$$
\begin{aligned}
& x(t)=x_{0} \cos \left(\kappa^{\prime} t+\psi\right)+\lambda \exp (k t) \\
& y(t)=\xi x_{0} \sin \left(\kappa^{\prime} t+\psi\right)+\lambda \xi^{\prime} \exp (k t) \\
& z(t)=z_{0} \cos \left(v^{\prime} t+\phi\right)
\end{aligned}
$$

where $x_{0}, z_{0}, \psi, \phi$ and $\lambda$ are free parameters. (As with any unstable equilibrium when there is no dissipation, there is also an exponentially decaying solution, which we have ignored. It corresponds to the stable invariant manifold of the epicyclic motion, and is of no practical importance, being of measure zero.)

As already mentioned the starting configuration is a homogeneous distribution of particles in an ellipsoid with axes $a, \xi a$ and $a$. To achieve the correct spatial distribution we choose the parameters $x_{0}$ and $z_{0}$ according to the following distributions

$$
\begin{aligned}
& F\left(x_{0}\right)=1-\left(1-\frac{x_{0}^{2}}{a^{2}}\right)^{3 / 2}, \\
& F\left(z_{0}\right)=1-\sqrt{1-\frac{z_{0}^{2}}{a^{2}-x_{0}^{2}}},
\end{aligned}
$$

with $x_{0} \in[0, a]$ and $z_{0} \in\left[0, \sqrt{a^{2}-x_{0}^{2}}\right]$. The parameters $\psi$ and $\phi$ are chosen randomly in the interval $[0,2 \pi]$.

Our recipe for constructing this model is given by Eqs. (15)-(17) (with three similar equations for the initial velocities and adopting $\lambda=0$ for the initial setup) and Eqs. (18) and (19). Here we show that this gives rise to the desired uniform space density.

From Eqs. (18) and (19) it follows that the joint probability density of the positive quantities $x_{0}, z_{0}$ is

$f\left(x_{0}, z_{0}\right)=\frac{3 x_{0} z_{0}}{a^{3}}\left(a^{2}-x_{0}^{2}-z_{0}^{2}\right)^{-1 / 2}$,

over the domain $x_{0}>0, z_{0}>0, x_{0}^{2}+z_{0}^{2}<a^{2}$. We also take the distributions of $\psi, \phi$ to be independent and uniform on $[0,2 \pi]$. The probability density of $x, y, z$ is therefore

$$
\begin{aligned}
f(x, y, z)= & \frac{3}{4 \pi^{2} a^{3}} \int x_{0} z_{0}\left(a^{2}-x_{0}^{2}-z_{0}^{2}\right)^{-1 / 2} \\
& \times \delta\left(x-x_{0} \cos \psi\right) \delta\left(y-\xi x_{0} \sin \psi\right) \\
& \times \delta\left(z-z_{0} \cos \phi\right) \mathrm{d} x_{0} \mathrm{~d} z_{0} \mathrm{~d} \psi \mathrm{d} \phi .
\end{aligned}
$$

The integration can be readily carried out by thinking of $x_{0}$, $\psi$ as a pair of plane polar coordinates and transforming to the corresponding Cartesian coordinates; similarly with $z_{0}, \phi$. The result is $f(x, y, z)=3 /\left(4 \pi \xi a^{3}\right)$, which is just the inverse of the volume of an ellipsoid with semi-axes $a, \xi a, a$, as required.

Since the evolution of position with time is just a translation in $\psi$ and $\phi$, it is clear that the space density remains uniform on this ellipsoid.

The above coordinates and velocities are defined in the cluster-centred frame which moves around the galaxy. To transform to the galactocentric rest-frame, we use the equations

$$
\begin{aligned}
& X(t)=(D+x) \cos (\Omega t)+y \sin (\Omega t) \\
& Y(t)=-(D+x) \sin (\Omega t)+y \cos (\Omega t) \\
& Z(t)=z \\
& \dot{X}(t)=(\dot{x}+y \Omega) \cos (\Omega t)+(\dot{y}-(D+x) \Omega) \sin (\Omega t) \\
& \dot{Y}(t)=-(\dot{x}+y \Omega) \sin (\Omega t)+(\dot{y}-(D+x) \Omega) \cos (\Omega t) \\
& \dot{Z}(t)=\dot{z} .
\end{aligned}
$$

To ensure that the initial distribution is not bound in the usual sense, its density must lie below a certain critical density. Roughly speaking this is the density $\rho_{0}$ of the background, i.e. the density which causes the galactic potential, and so

$$
\rho<\frac{V_{0}^{2}}{4 \pi G D^{2}}=\rho_{0}
$$

(cf. Eq. (12)).

The simulations were performed with the particle-mesh code SUPERBOX (Fellhauer et al. 2000) with 1000000 particles and meshes with $n=128$ grid points per dimension, to achieve high resolution. We chose the following grid sizes for the three nested grids which the code uses: $R_{\text {system }}=12 \mathrm{kpc}$, $R_{\text {out }}=2 \mathrm{kpc}$ and $R_{\text {core }}=0.4 \mathrm{kpc}$. As the resolution of SUPERBOX is given by the length of one grid cell, these parameters give a resolution of $6.45 \mathrm{pc}\left(\right.$ res $\left.=2 R_{\text {core }} /(n-4)\right)$ in the central region of the particle distribution. The code has a simple leapfrog integration scheme with fixed timestep, which in our simulations was DT $=0.1 \mathrm{Myr}$. To ensure that our conclusions do not depend on the parameters of the code, we repeated one simulation with lower resolution and lower particle number, and found no significant change in the results.

\section{Results}

Using initial conditions specified in the previous section, we have conducted a parameter survey for various choices of the semi-minor axis $a$ and the density $\rho$ (Table 1 ). The distance to the galactic centre and the circular velocity were held at $D=$ $10 \mathrm{kpc}$ and $220 \mathrm{kms}^{-1}$, respectively.

\subsection{Lifetime of the systems}

In every simulation which satisfied Eq. (28) initially, the distribution was quite stable for several orbits around the galactic centre. Figure 2 shows the Lagrangian radii of the simulation where $a=50 \mathrm{pc}$ and $\rho / \rho_{0}=0.1$. The orbital period around the 
Table 1. Parameter space of the simulations. Each simulation performed is marked with a bullet point.

\begin{tabular}{rrrrrrrrr}
\hline \hline$a$ & & & \multicolumn{8}{c}{$\rho / \rho_{0}$} & & & \\
{$[\mathrm{pc}]$} & 0.01 & 0.05 & 0.10 & 0.50 & 1.00 & 2.00 & 5.00 & 10.00 \\
\hline 10 & & & $\bullet$ & & & & & \\
50 & $\bullet$ & $\bullet$ & $\bullet$ & $\bullet$ & $\bullet$ & $\bullet$ & $\bullet$ & $\bullet$ \\
100 & & & $\bullet$ & & & & & \\
500 & $\bullet$ & $\bullet$ & $\bullet$ & $\bullet$ & $\bullet$ & & $\bullet$ & $\bullet$ \\
1000 & $\bullet$ & $\bullet$ & $\bullet$ & $\bullet$ & $\bullet$ & & $\bullet$ & $\bullet$ \\
5000 & & & $\bullet$ & & & & & \\
\hline
\end{tabular}

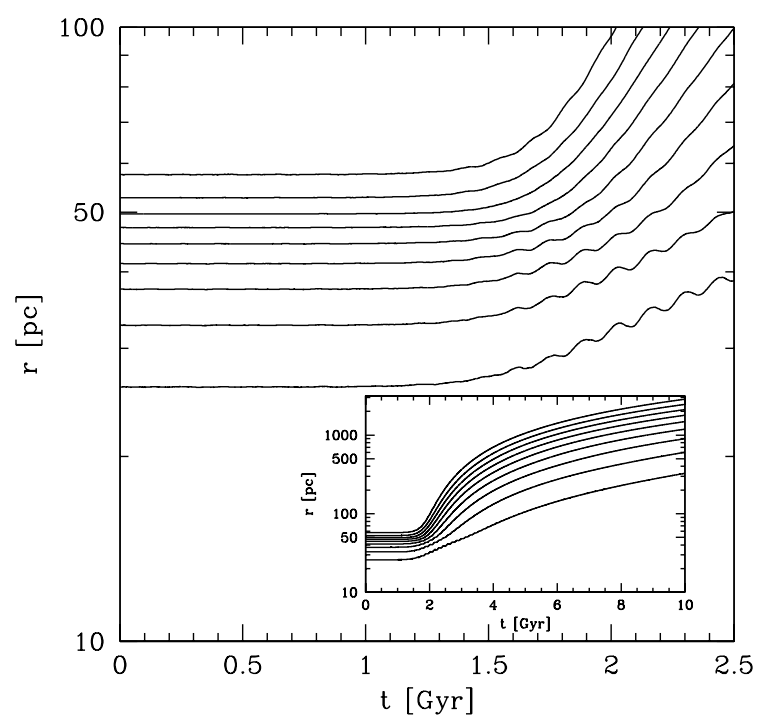

Fig. 2. Lagrangian radii $(10,20, \ldots, 90 \%)$ for the simulation with $a=50 \mathrm{pc}$ and $\rho / \rho_{0}=0.1$. The large figure shows the time interval during which the distribution is stable; the small figure shows the whole simulation.

galaxy in our simulations was about 280 Myr. Clearly the distribution did not evolve much for almost 6 revolutions around the galactic centre.

Because this is an unbound configuration there is no conventional definition for its time of dissolution. For the purpose of comparing the simulations, we adopt the time, $t_{\text {diss }}$, when

$r_{\mathrm{h}}=1.5 r_{\mathrm{h}, 0}$

where $r_{\mathrm{h}}, r_{\mathrm{h}, 0}$ denote the current and initial half-mass radii, respectively. With this definition, the dissolution time is shown in Fig. 3 as a function of the density, for different values of $a$. When $\rho$ is negligible, the particles describe independent epicycles with a common guiding centre, and so $t_{\text {diss }}$ is essentially infinite (in the epicyclic approximation).

Except at the highest densities the results can be fitted approximately with power laws

$t_{\text {diss }}=A\left(\frac{\rho}{\rho_{0}}\right)^{-\gamma}$.

Best-fitting values for the parameters are shown in Table 2.

We have shown in Eq. (13) that the exponent of the exponential growing solution, $k$, is approximately equal to the square root of $B_{3}$ which itself is proportional to the squareroot of the density. This approximation is valid for very small

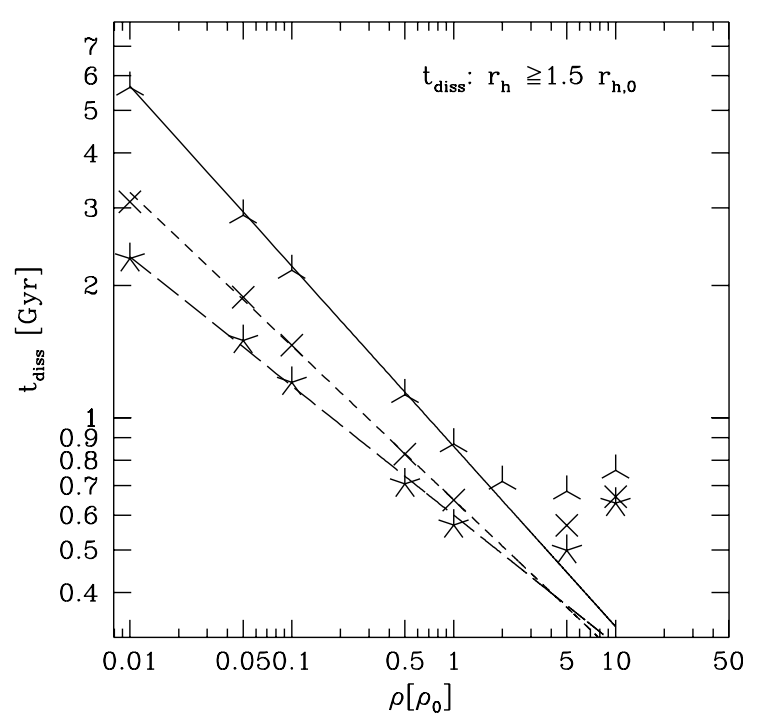

Fig. 3. Dissolution time as a function of the initial density of the particle distribution for different values of $a$. Three-pointed stars correspond to $a=50 \mathrm{pc}$, crosses to $a=500 \mathrm{pc}$ and five-pointed stars to $a=1000 \mathrm{pc}$. The dissolution time is a decreasing function of $\rho$, except for $\rho / \rho_{0}>1.0$. In this case the distribution is indeed unstable (according to our criterion), but before expanding it undergoes a slight initial collapse which leaves behind a small bound core.

Table 2. Fitting parameters for the power law relating the dissolution time to the initial density of the system.

\begin{tabular}{rcc}
\hline \hline$a[\mathrm{pc}]$ & $A[\mathrm{Gyr}]$ & $\gamma$ \\
\hline 50 & 0.86 & 0.41 \\
500 & 0.65 & 0.35 \\
1000 & 0.60 & 0.29 \\
\hline
\end{tabular}

initial density. If, however, we solve Eq. (13) numerically over the range of densities corresponding to our simulations, and fit the results by a power law, we obtain approximately

$k \propto \rho^{0.40 \pm 0.03}$.

This shows that, as long as the size $a$ of the system is sufficiently small that the epicyclic approximation holds, the dissolution time should be almost proportional to $\rho^{-0.4}$. This is confirmed by Table 2 .

The effect of the exponentially growing term can be clearly seen in Fig. 4, which shows particle orbits from the simulation with $a=50 \mathrm{pc}$ and $\rho / \rho_{0}=0.1$ up to about $t_{\text {diss. }}$. Evidently, the exponential instability is much stronger in the $y$-coordinate than in the $x$-coordinate; this is to be expected, by Eq. (14). The result is that the expansion of the system takes place mainly in the direction along the orbit around the galactic centre.

While Fig. 4 shows the effect of the exponential instability on individual orbits, Fig. 5 shows its influence in the expansion of the system as a whole. At very early times it is hidden in the random noise of the simulation, or indeed by the epicyclic motion itself. Its presence is most clear at intermediate times; later on, when the self-gravity of the system becomes negligible, the expansion is sub-exponential.

We also investigated whether there is a relation between the size of the system and the time of dissolution, but as one can 

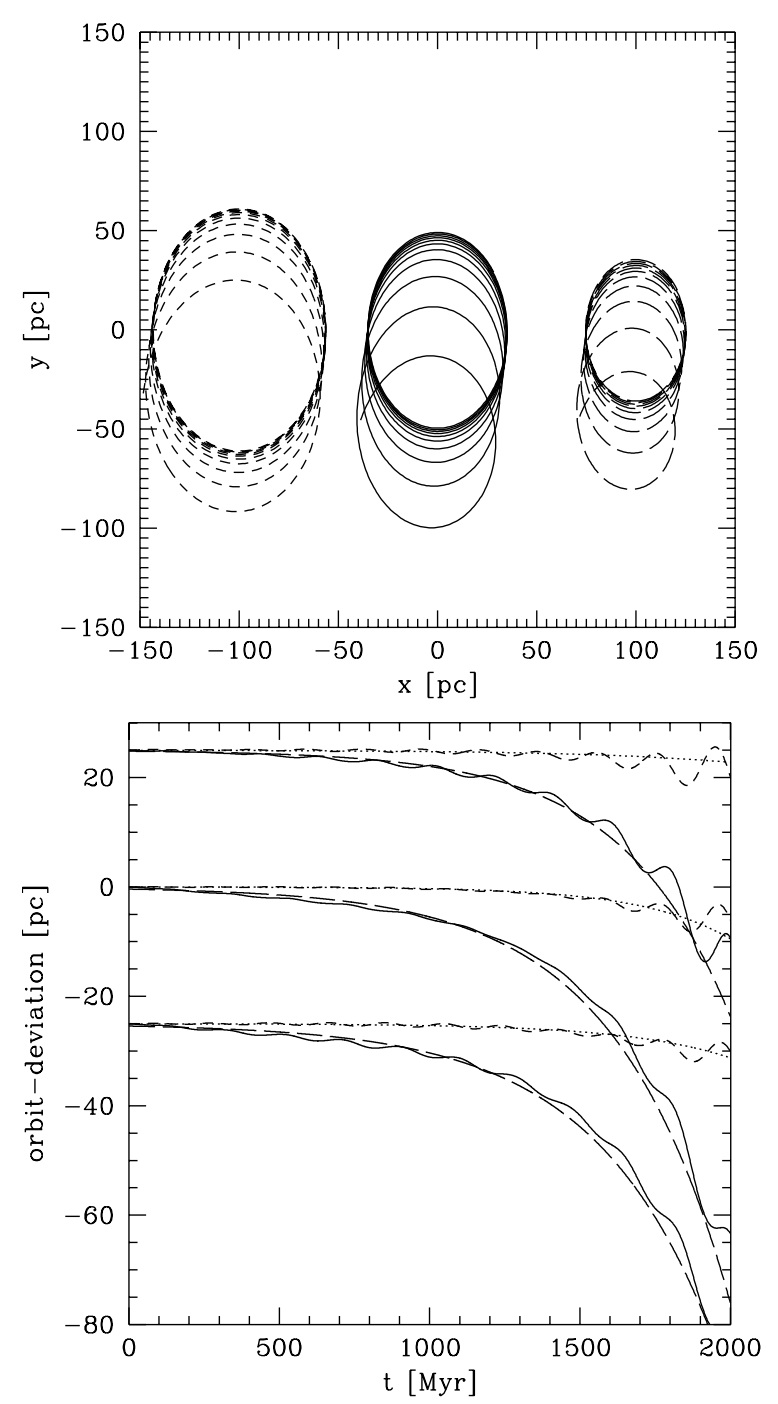

Fig. 4. Upper: orbits (in the co-moving cluster frame, for $t \leq 2 \mathrm{Gyr}$ ) of three particles in the simulation with $a=50 \mathrm{pc}$ and $\rho / \rho_{0}=0.1$. For clarity two of the orbits have been shifted in the $x$-direction by $\pm 100 \mathrm{pc}$. One clearly sees the growing deviation from simple epicyclic motion. Lower: $x$ (short dashed) and $y$ (solid) residuals (i.e. coordinates with the epicyclic solution subtracted) for the above three particles as functions of time. The dotted $(x)$ and long dashed $(y)$ lines show fitting functions for an exponential growing solution. Again for clarity the curves of two particles are shifted by $\pm 25 \mathrm{pc}$.

see in Fig. 6 there is no obvious analytical relation. The fact that small systems ( $\operatorname{small} a$ ) have longer dissolution times than large systems can be understood by noting that the epicyclic approximation becomes less accurate for larger systems.

\subsection{Appearance of the systems}

Though the initial conditions are highly idealised, it is interesting to look briefly at how the appearance of such a system evolves as it disperses. Even after the system starts to disperse, forming tidal tails along its orbit, we still find a measurable density enhancement at the position of the unbound cluster. In Fig. 7 we show the measured surface density in one of the runs at $t=1,5$ and 10 Gyr. The surface density distribution
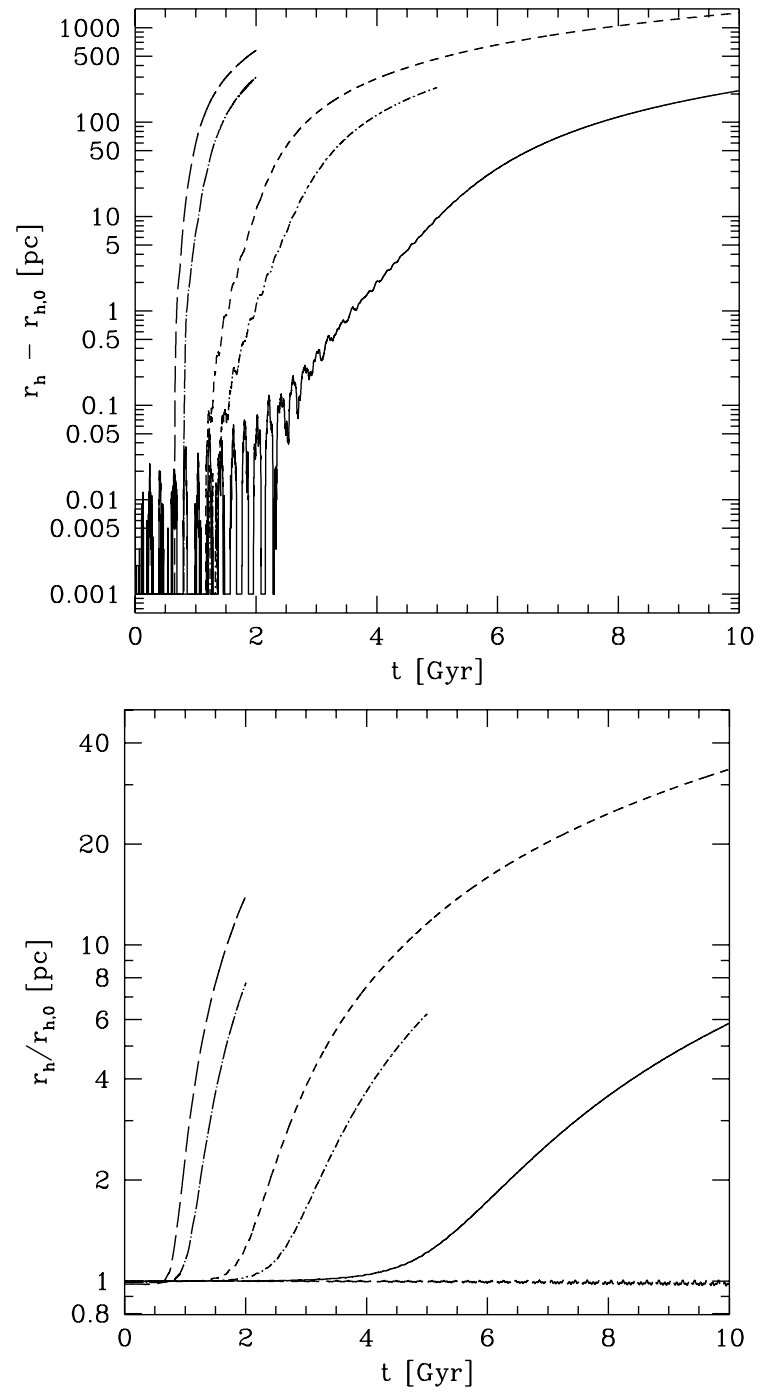

Fig. 5. Upper: difference between the current half-mass radius $r_{\mathrm{h}}$ and the half-mass radius at the beginning of the calculations $r_{\mathrm{h}, 0}$. All values are taken from simulations with $a=50 \mathrm{pc}$ and varying density (from left to right: 1.0, 0.5, 0.1, 0.05 and 0.01 times the background density). Lower: ratio of $r_{\mathrm{h}}$ and $r_{\mathrm{h}, 0}$ for the same calculations. (The almost horizontal plot is a test calculation in which self-gravity was neglected in both the initial conditions and in the run. For this model the only evolutionary mechanism is the inexactness of the epicyclic approximation.) The phase of exponential growth is clearly visible. If the half-mass radius gets very large then the self-gravity of the system is negligible, and one sees the effects of epicyclic drift: the curves change to a linear growth.

resembles that of an object with a large core radius. At late times the surface density is almost flat until nearly $100 \mathrm{pc}$ and then it turns over to a shallow power law with an index of approximately -1.6 . Fitting an exponential gives an exponential scale-length of approximately $100 \mathrm{pc}$.

The kinematics of the model depend on the frame in which it is viewed, and are complicated by its initial rotation. At $t=0$ it can be seen, by setting $\lambda=0$ in Eqs. (15)-(17), differentiating, and setting $t=0$, that $\dot{x}=-\kappa^{\prime} y / \xi, \dot{y}=\xi \kappa^{\prime} x$ and $\dot{z}=-z_{0} v^{\prime} \sin \phi$. Thus along the $x$ - and $y$-directions the system appears to rotate, though with different angular velocities. 


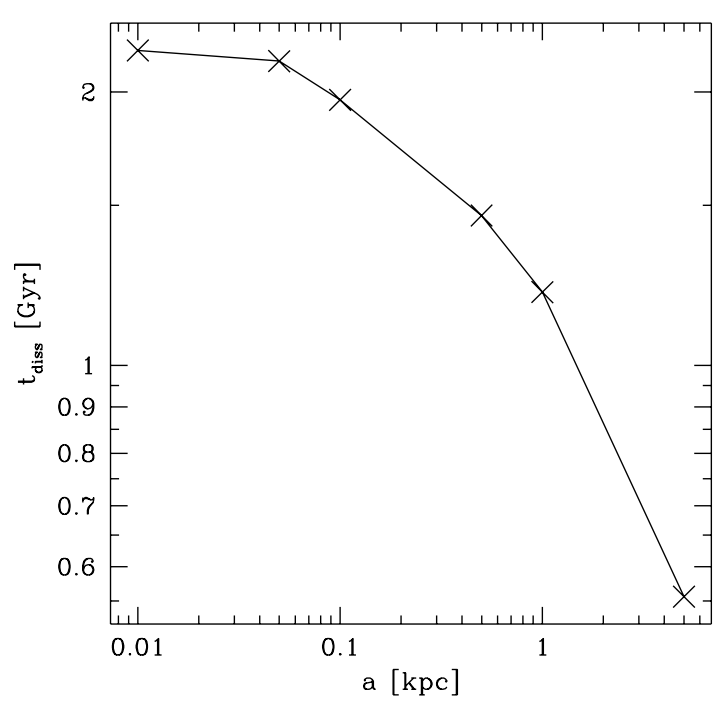

Fig. 6. Dissolution time as a function of the size $a$ of the system. The density is kept constant at $\rho / \rho_{0}=0.1$.

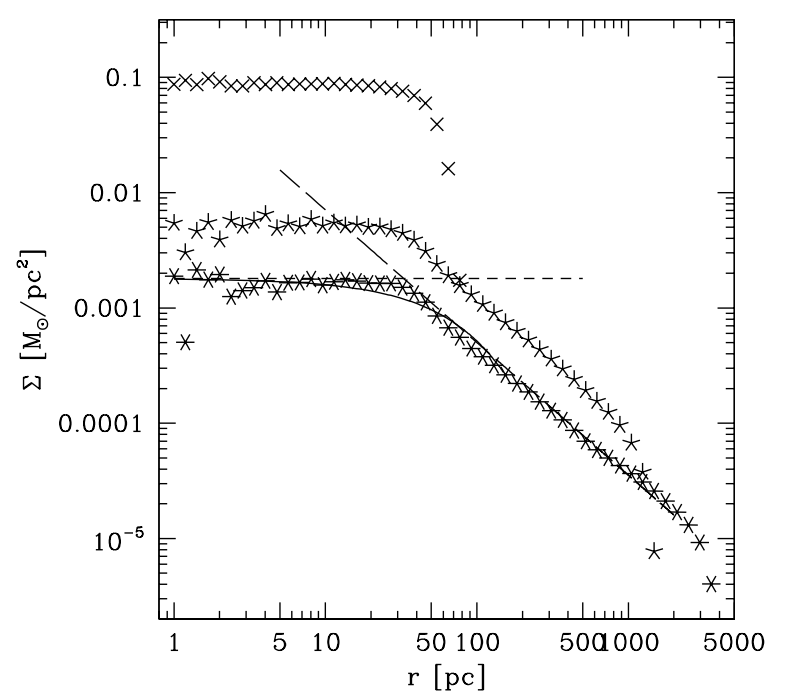

Fig. 7. Surface density profile of the simulation with $a=50 \mathrm{pc}$ and $\rho / \rho_{0}=0.1$ at times $t=1 \mathrm{Gyr}$ (crosses), $t=5 \mathrm{Gyr}$ (five-pointed stars) and $t=10 \mathrm{Gyr}$ (six-pointed stars) measured in concentric radial bins centred on the object and looking along the $Z$-axis. Also shown are the fitting lines for the $t=10 \mathrm{Gyr}$ data. Short dashed line is the horizontal fit for the inner part, long dashed line the power law fit for the outer part and the solid curve is an exponential fit.

In the $z$-direction the velocity dispersion, $\sigma_{z}$, follows the dispersion of $z_{0}$, i.e. the thickness in the $z$-direction. We also see that, at a given distance $r_{x}$ from the $x$-axis, the dispersion in $\dot{x}$ is given by $\sigma_{x}^{2}=\left\langle\dot{x}^{2}\right\rangle=\kappa^{\prime 2}\left\langle y^{2}\right\rangle / \xi^{2}=\kappa^{\prime 2} r_{x}^{2} /\left(2 \xi^{2}\right)$, with a similar result for $\sigma_{y}^{2}$. As the time of disruption (as defined here) is approached, the profiles of $\sigma_{x}$ and $\sigma_{y}$ approach that for $\sigma_{z}$, which itself changes little from that at $t=0$. The evolution of $\sigma_{x}$ is faster, however, because the orbits diffuse transverse to this line of sight more quickly (Fig. 4).

In the galactocentric frame at $t=0$ one sees from Eqs. (22)-(27) that the corresponding results are $\dot{X}=Y\left(\Omega-\kappa^{\prime} / \xi\right)$ and $\dot{Y}=-\Omega D+(X-D)\left(-\Omega+\xi \kappa^{\prime}\right)$, while $\dot{Z}=\dot{z}$. Again there appears to be rotation (with different angular velocities) in the $X$ and $Y$ directions, but for $\dot{X}$ it can be shown from Eqs. (4) and (11) that the angular velocity vanishes if $\rho=0$. As with the velocity dispersions in the rotating frame, we find that all three velocity dispersions become comparable as the time to disruption approaches. Beyond the original radius of the system, however, the profiles of $\sigma_{x}$ and $\sigma_{y}$ increase with projected radius, because of galactic rotation.

The system which is shown in Fig. 7 has a total initial mass of about $660 M_{\odot}$. At $t=10 \mathrm{Gyr}$ the central line-of-sight velocity dispersion is about $1 \mathrm{~km} \mathrm{~s}^{-1}$ and the core radius is approximately $100 \mathrm{pc}$. If one followed the arguments of Mateo (1998) to calculate the virial mass (assuming the object is bound), using his formula

$M_{\mathrm{vir}} \approx 167 \beta r_{\mathrm{core}} \sigma_{\text {los }}^{2}$,

with $\beta=8$, the result is roughly $10^{5} M_{\odot}$.

\section{Conclusions and discussion}

We have studied the evolution of a special class of unbound systems orbiting at a constant distance from the centre of an isothermal galaxy model. Initially the systems have uniform density within an ellipsoidal region. They move according to epicyclic theory (modified for the self-gravity of the system), with guiding centre at the centre of the ellipsoid. These systems are exact self-consistent equilibrium models within a steady tidal field.

While these equilibrium models are believed to be new in the context of a tidally limited stellar system, they have properties in common with Freeman's models of a barred galaxy (Freeman 1966; Binney \& Tremaine 1987). There too the effective potential inside the system is quadratic in a uniformly rotating frame. Freeman's models are the two-dimensional limit of a flattened ellipsoid. It would be interesting to explore this link further.

Our simulations show that, depending on their initial density and radius, the systems we have constructed survive with only modest expansion for a surprisingly long time. When our results are scaled to a circular orbit of radius $10 \mathrm{kpc}$ with a circular velocity of $220 \mathrm{kms}^{-1}$, a system of semi-minor axis $50 \mathrm{pc}$ and density 0.2 times that of the background galaxy survives for up to several Gyr (i.e. about 20 Galactic orbits). For small systems the dependence on initial density, and the direction in which the dispersal takes place, can be understood on the basis of the modified epicyclic theory. For large systems the lifetime may be limited by the accuracy of epicyclic theory. Even though the systems gradually disperse, there are measurable density enhancements even after a Hubble-time.

Our results have interesting parallels with those of Kleyna et al. (2003), even though the initial conditions and galactic field are completely different. They investigated the survival of an unbound clump of particles (with an initial Gaussian velocity distribution) in the inner part of the dark halo of a dwarf satellite. As long as the dark matter halo is not cusped, they too found that the system disperses only on long time-scales (longer than a Hubble-time). The longevity of their models relies partly on the relatively cold initial conditions and partly on the near-harmonic potential of the core of the host galaxy. 
Our models are also cold initially (in the sense that the velocity dispersion in $x$ - and $y$ - at a given point is zero), and the potential is approximately harmonic inside the model (Eqs. (6)-(8)).

Idealised models, such as those discussed in this paper, have the advantage that they are suitable for analytical study, which can provide greater understanding than the compilation of results from simulations. An example of this is our result on the dependence of dissolution time on density. The drawback of idealised models is that the results cannot be applied directly to real systems, and can only be suggestive. On the other hand a study of Fig. 2 suggests that the role of the special initial conditions may be confined to the phase in which the Lagrangian radii do not evolve by a large factor. The subsequent evolution (e.g. as shown in late-time plots in Fig. 7) may be applicable more widely.

The systems in nature which most closely correspond to ours are open clusters. Shortly after the tidal radius of an evolving cluster has shrunk to zero, we expect that the remnant consists of an inhomogeneous spatial distribution of stars with a preponderance of retrograde rotation, in nearly circular motion in the disc of the galaxy. Our models are initially homogeneous, with pure retrograde rotation. They nevertheless suggest that it would be interesting to perform similar simulations with more realistic initial conditions. If these also exhibited the long dissolution times we find for some models, this would suggest that it may be possible to detect the remnants of dissolved star clusters even now.

Other subsystems within galaxies (satellite galaxies and globular clusters) differ further. Their galactic orbits are not nearly circular, as a rule, and the resulting time-dependent tide presumably causes faster dissolution, which is enhanced also by disk shocking. For this reason, we consider that the dissolution time we find (when suitable scaled to the orbital period) is an upper limit to the lifetime of such systems.

Acknowledgements. M.F. acknowledges financial support through DFG-grant FE564/1-1 and KR1635/5-1. We want to thank R. Spurzem, C. Theis and P. Kroupa for fruitful discussions of the results. We thank R. H. Miller for suggesting the connection with Freeman's models.

\section{References}

Baumgardt, H. 1998, A\&A, 330, 480

Baumgardt, H., \& Makino, J. 2003, MNRAS, 340, 227

Binney, J., \& Tremaine, S. 1987, Galactic Dynamics (Princeton University Press)

Chandrasekhar, S. 1942, Principles of Stellar Dynamics (University of Chicago Press)

Fellhauer, M., Kroupa, P., Baumgardt, H., et al. 2000, New Astron., 5, 305

Fellhauer, M., Baumgardt, H., Kroupa, P., \& Spurzmen, R. 2002, CeMDA, 82, 113

Freeman, K. C. 1966, MNRAS, 134, 17

Giersz, M., \& Heggie, D. C. 1997, MNRAS, 286, 709

Hénon, M. 1969, A\&A, 1, 223

Kleyna, J. T., Wilkinson, M. I., Gilmore, G., \& Evans, N. W. 2003, ApJ, 588, L21

Mateo, M. 1998, ARA\&A, 36, 435

Velazquez, H., \& White, S. D. M. 1999, MNRAS, 304, 254 